\title{
Frontal Cortical Kindling in Cats
}

\author{
AKIRA WAKE AND JUHN A. WADA
}

SUMMARY: Our observation of amygdaloid seizure development in cats indicated early afterdischarge propagation into basal cortical areas prior to the development of bifrontal sharp theta discharge. In view of the implied participation of the frontal lobe in amygdaloid kindling, both fractional lesioning and kindling of selective areas were performed. This paper summarizes our stimulation study involving premotor, prefrontal, mesial frontal and orbital cortices. Except for the orbital series which showed a rather strikingly similar pattern of seizure development to that of amygdaloid kindling, all areas showed significantly different features in terms of the speed of seizure development, afterdischarge propagation, fragility of developing seizure, final stage 5 seizure and post-ictal behavior pattern, interictal discharge morphology and propagation, and generalized seizure triggering threshold intensity. All these findings suggest that the frontal lobe participates in, but is not essential for, the amygdaloid seizure development. The results of ongoing fractional lesion series support such a conclusion.

RÉSUMÉ: Plusieurs séries d'expériences impliquant des lésions partielles, et le "kindling" de régions sélectives du cerveau, indiquent que le lobe frontal participe dans le développement de la convulsion amygdaloidienne, mais qu'il n'est pas essentiel.

From the Division of Neurological Sciences. Faculty of Medicine, University of British Columbia, Vancouver, Canada.

This work is supported by grants from Canadian Medical Research Council and U.S. National Institute of Neurological, Communicative Disease and Stroke awarded to J. A. Wada.

Reprint requests to D. A. Wake, Health Sciences Center Hospital, 2075 Wesbrook Place, Vancouver, B.C. V6T IW5 Canada.
Appropriately spaced electrical brain stimulation which may be initially ineffective will result in a progressive electroclinical seizure development with persistent manifestations of widespread functional reorganization of brain (Goddard et al., 1969). The most productive area of investigation in this "kindling" phenomenon involves deep limbic structures in rats (Goddard, 1967; Racine, 1972 a \& b; Corcoran, 1973), cats (Delgado \& Savilano, 1961; Goddard, 1969; Morrell, 1973; Wada, 1974), rhesus monkeys (Goddard, 1969; Wada, 1974) and baboons (Wada \& Osawa, 1974). Early study of Goddard et al (1969) suggested that the kindling effect may be obtained from frontal cortex in rats, which has now been confirmed by a recent study detailing the differential characteristics of kindling obtained from this neocortical site and amygdala in rats (Racine, 1975).

In our previous study of amygdaloid kindling in rats, cats and baboons (Wada, Sato \& McCaughran, 1974), we found that progressive involvement of basal cortical areas as well as the development of theta discharge in the frontal cortex were associated with complex seizure patterns, while increasing complexity of spikeness in the rolandic cortex was associated with the onset of focal motor events. In order to assess the possible role of the frontal lobe in the amygdaloid seizure development, a series of studies employing both fractionated lesion and stimulation of selective areas of the frontal lobe was performed. This paper summarizes the results of our stimulation study involving prefrontal, premotor, mesial frontal, and orbital cortices in cats. The findings suggest that the frontal lobe participates in, but is not essential for, the amygdaloid seizure development.

\section{MATERIALS AND METHODS}

Eight cats, $3.0-4.7 \mathrm{~kg}$, were implanted with permanent electrodes bilaterally into the lateral amygdala (AM), pyriform cortex, (PYR), hippocampus, (HIPP), midbrain reticular formation (MRF) globus pallidus (GP), and respective frontal or orbital cortical areas (F). Bipolar recording electrodes were made by attaching a stainless steel wire $0.19 \mathrm{~mm}$ in diameter to No. 30 stainless steel tubing, insulated except for tips, with interelectrode distance of 1 $\mathrm{mm}$. The stimulating electrode was the same stainless steel wire, insulated except for $1 \mathrm{~mm}$ at the tip, and was attached to the recording electrode.

Cortical electrodes were the same stainless steel wire insulated except for the tip. The exposed portion of the stimulating electrode was inserted perpendicularly into the desired cortical area and the recording electrode was resting on the cortical surface. Two animals each were used for stimulation of prefrontal. premotor, mesial frontal, and orbital cortices (Fig. 1). At least 2 weeks elapsed after surgery before commencement of cortical stimulation. Stimulation, delivered monopolarly to either left or right cortical site, was given once a day with $60 \mathrm{~Hz}$ sine wave lasting for one second. The intensity of the stimulus was regulated by a constant current unit. The stimulation began with $100 \mu \mathrm{A}$, followed by subsequent daily increases by $200 \mu \mathrm{A}$ steps until localized afterdischarge (AD) was elicited. On each subsequent day, the stimulus intensity was reduced with $50 \mu \mathrm{A}$ steps until $\mathrm{AD}$ was no longer elicited. The last intensity at which AD was produced was arbitrarily designated as AD threshold. Daily stimulation was carried out between 9.00 a.m. and noon. 
The animals were placed in the observation chamber $(46 \times 90 \times 65$ $\mathrm{cm})$ with a one way mirror. The EEG was recorded on a 10 channel Grass machine. Stimulation at AD threshold was delivered at least 10 15 minutes after the animal assumed a resting posture subsequent to the beginning of EEG recording within the chamber. When the final stage of generalized convulsive seizure developed in an animal, the stimulation was repeated for 5 more days. Then, the stimulus intensity was gradually reduced until the animal failed to respond with a generalized convulsive seizure. The stimulus intensity on the last day when the latter was induced was arbitrarily designated as the generalized seizure triggering threshold (GST). All the animals were sacrificed under deep anesthesia and their brains were perfused with physiological saline and $10 \%$ formalin. The tissue was frozen, serially sectioned $(60 \mu)$ and stained with cresyl violet for the identification of the electrode tips. The recording electrodes were in the intended structures and the stimulating electrodes were localized in respective cortical areas (Fig. 1).

\section{RESULTS}

Findings are summarized in Tables 1 and 2 and details are given below.

The AD threshold was $100-400$ $\mu \mathrm{A}$ and the average number of daily electrical stimulations required for the development of a final stage convulsion was 36 (9-91). There was

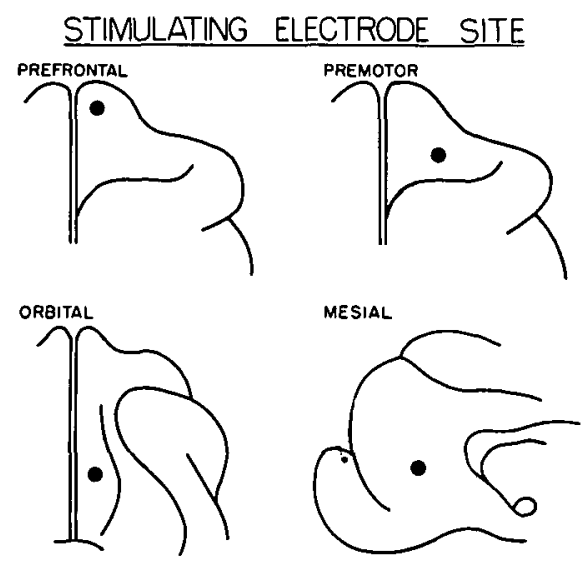

Figure 1 -Localization of stimulating electrode sites.
TABLE 1

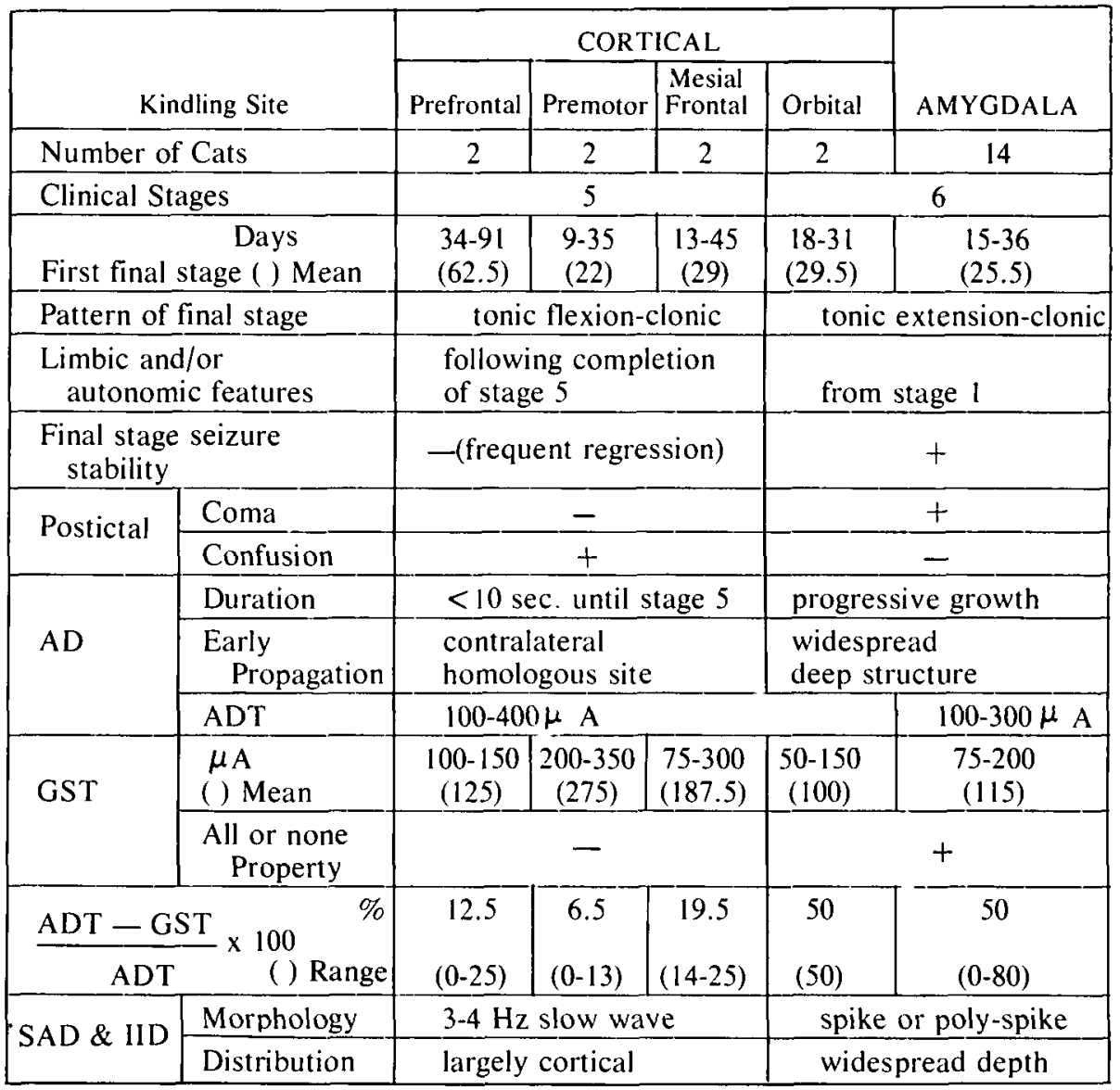

AD: Afterdischarge; ADT: Afterdischarge threshold; GST: Generalized seizuretriggering threshold; SAD: Secondary afterdischarge; IID: Interictal discharge. no clear relationship between the number of stimulations required for completion of kindling and the absolute intensity of the AD threshold. For example, cat 621 was stimulated in the premotor cortex at $400 \mu \mathrm{A}$ and required 35 days, whereas cat 634 was stimulated in premotor cortex at $200 \mu \mathrm{A}$ and required only 9 stimulations.

\section{TABLE 2}

\begin{tabular}{|c|c|c|c|c|c|}
\hline \multirow{2}{*}{$\begin{array}{l}\text { Kindling } \\
\text { Site }\end{array}$} & \multicolumn{4}{|c|}{ CORTICAL } & \multirow{2}{*}{ AMYGDALA } \\
\hline & Prefrontal & $\begin{array}{l}\text { Mesial } \\
\text { Frontal }\end{array}$ & Premotor & Orbital & \\
\hline I & \multicolumn{4}{|c|}{$\begin{array}{c}\text { Contralateral Ipsilateral } \\
\text { Head turning }\end{array}$} & $\begin{array}{l}\text { Ipsilateral } \\
\text { Facial twitching }\end{array}$ \\
\hline II & \multicolumn{3}{|c|}{ Rhythmic mouth movement } & & $\begin{array}{l}\text { Bilateral } \\
\text { Facial twitching }\end{array}$ \\
\hline III & \multicolumn{3}{|c|}{ Rhythmic head turning } & \multicolumn{2}{|c|}{ same to $\longrightarrow$ Head nodding } \\
\hline IV & \multicolumn{3}{|c|}{$\begin{array}{l}\text { Contralateral } \\
\text { Hemiconvulsion }\end{array}$} & & $\begin{array}{l}\text { Contralat. forepaw } \\
\rightarrow \text { tonic, Contralat. } \\
\text { head turning \& circling }\end{array}$ \\
\hline V & \multicolumn{3}{|c|}{ Generalized Convulsion } & & $\begin{array}{l}\rightarrow \text { Clonic jerking while } \\
\text { standing }\end{array}$ \\
\hline VI & & & & & $\rightarrow$ Generalized convulsion \\
\hline
\end{tabular}


Clinical Seizure Development:

The chronological pattern of seizure development was classified into the following five stages and representative examples are given in Figure 2.

Stage 1. Head turning. The direction of this head turning was ipsilateral in premotor and orbital animals and contralateral in prefrontal and mesial animals. This was characterized by turning of the head postero-laterally as though to look up and back and maintaining this posture for a few more seconds after the termination of the AD. There was no eye deviation or change of pupillary size. The extent of the head turning was greatest in the prefrontal followed by mesial and premotor groups in that order, and was least in the orbital group.

Stage 2. Bilateral perioral twitching and blinking. This was characterized by the development of sniffing-like movement around nose which eventually became bilateral perioral twitching. Except for the orbital group, all the animals showed progression of this twitching to involve the whole face, and it was therefore associated with bisymmetrical rhythmic blinking.

During Stage 2, the orbital group began to develop unique clinical manifestations which separated it from the rest of the animals. These were pupillary dilatation, chewing, salivation and urinary incontinence. In addition, there was a development of predominantly ipsilateral rhythmic facial twitching. The pattern of subsequent clinical seizure development in the orbital group was identical to that of AM kindling with head nodding, contralateral head turning, rapid circling and clonic jumping while standing before falling down with the generalized convulsion (C). The rest of the animals showed the following successive stages.

Stage 3. Rhythmic head turning. At this stage the degree of head turning became maximal to the extent that the animal was now looking almost directly backward. However, in contrast to the sustained nature of head turning in earlier stages, there was now a slow rhythmic interruption as though the animal was intermittently trying to restore normal head position.

\section{Stage 4. Contralateral hemiconvul-} sion beginning with tonic extension of contralateral forepaw. The animals began to circle contralaterally as the degree of head turning intensified (in prefrontal and mesial group) or turn to face the front (premotor groups). There was subsequent tonic extension and jerking of contralateral forepaw followed by the same in the contralateral hindpaw resulting in a hemiconvulsion. However, at this time the animals were still capable of maintaining the standing position.

Stage 5. Generalized clonic-tonicclonic seizure. As the clonic jerking became generalized, the animals lost postural control and fell abruptly with intense tonic flexion of neck and upper extremities but with moderate extension of hindlegs. This was followed by a secondary bisymmetrical clonic convulsion. When the Stage 5 seizure was repeated, some of the animals began to display chewing, salivation, pupillary dilatation and urinary incontinence during the latter part of the individual seizures. Postictally, all the animals (except for the orbital group which showed a profound comatose inactivity) quickly righted themselves and displayed a state of confusion for a prolonged period.

Some animals showed skipping of a stage or stages during their seizure development (Fig. 2, Cat No. 634 and No. 659) but these missed stages were always represented in a compressed manner in the sequential march of ictal events during the next stage seizure.

As shown in Figure 2, one of the outstanding features of the frontal cortical kindling was the instability of the final Stage 5 generalized convulsion. That is, except for one mesial animal, all the animals showed a frequent regression to earlier seizure stages even after Stage 5 had been reached. Although no systematic exploration was made, the semiperiodic tendency of regression suggested that if the stimulation had been spaced every 48 hours instead of 24 hours, they might have responded with more stable Stage 5 seizures. Similarly, if the animals were stimulated while asleep they tended to show relatively stable Stage 5 seizures. When all of the animals finally attained a stable Stage 5 seizure state on successive five days, the critical threshold required for the production of the

\section{CLINICAL SEIZURE DEVELOPMENT \& AD DURATION}
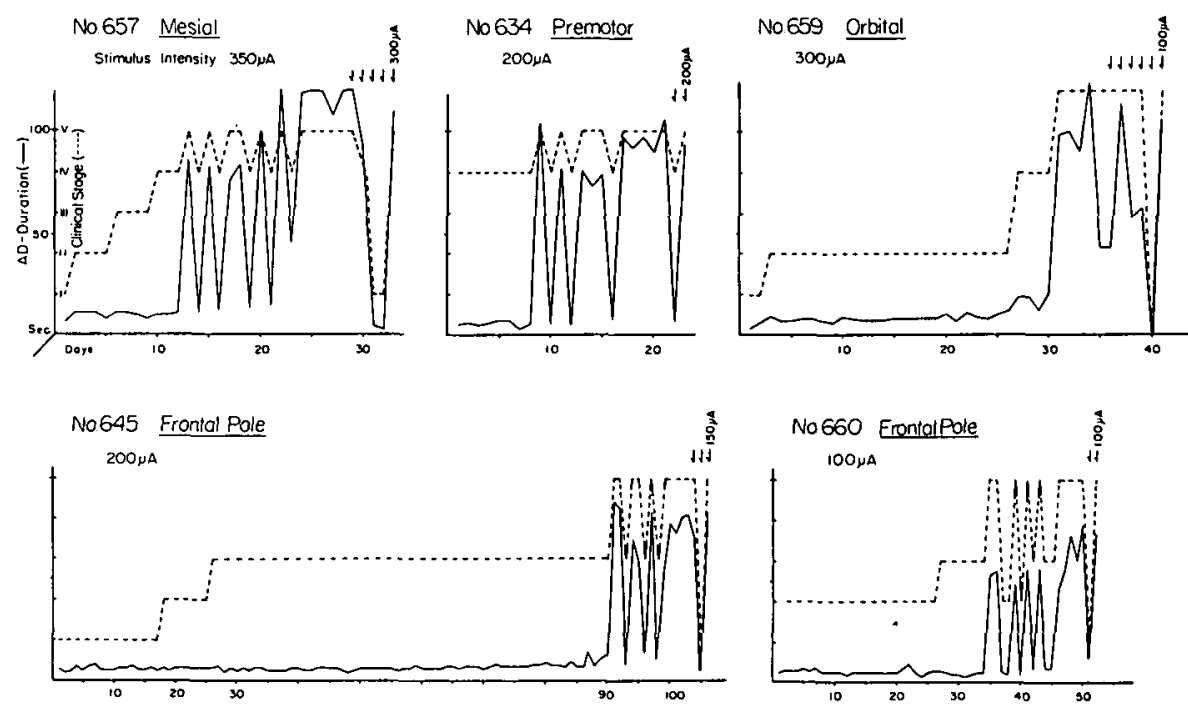

Figure 2-Chronology of electroclinical seizure development. Note instability of Stage 5 seizure with a tendency to 48 hour cycle. Note relatively clinical stability of orbital Stage 6. 
generalized convulsion (GST) was examined. Range of GST was 50-350 $\mu \mathrm{A}$. When compared with the initial $\mathrm{AD}$ threshold, this represented an average of $50 \%$ reduction in the orbital group in contrast to average of $13 \%(0-25)$ in all other animals.

\section{Afterdischarge $(A D)$ :}

(A) AD Duration: All the animals maintained a rather stable but brief AD duration ranging 5-10 sec. during Stages 1-4 but developed an explosive growth when the final Stage 5 was reached. Subsequently, the AD duration remained unchanged except for intermittent reduction at the time of regression to earlier clinical stages (Fig. 2).

(B) AD Propagation: During Stages 1-4, major AD propagation was observed into contralateral homologous cortical areas with less prominent $\mathrm{AD}$ projection into deep structures with abrupt generalization when the final stage was reached (Fig. 3). In contrast, the orbital group showed early AD propagation into $A M$ with a subsequent pattern of widespread subcortical dissemination identical to that of $\mathrm{AM}$ kindling (Fig. 4).

Termination of AD was simultaneous in all the structures throughout. It was noted that the emergence of self-sustained AD pattern at the hippocampus during the latter part of an indidividual seizure tended to coincide with the development of limbic-autonomic manifestations as well as relative stability of Stage 5 seizure.

(C) Secondary afterdischarge (SAD) and interictal discharge (IID): (Fig. 5). Within 10-30 sec. after the termination of the first cortical AD, high amplitude rhythmic 3-4 per sec. slow wave burst activity began to appear lasting for a few seconds at the stimulated cortical site (SAD). With repeated stimulation there was a gradual but progressive increase of its duration up to about 30 seconds with less prominent but synchronous activity of shorter duration in the contralateral homologous cortical site. This was followed by a frequent recurrence of short trains of similar burst activity lasting for about $1 \mathrm{sec}$ (IDD) and persisting for 24 hours.

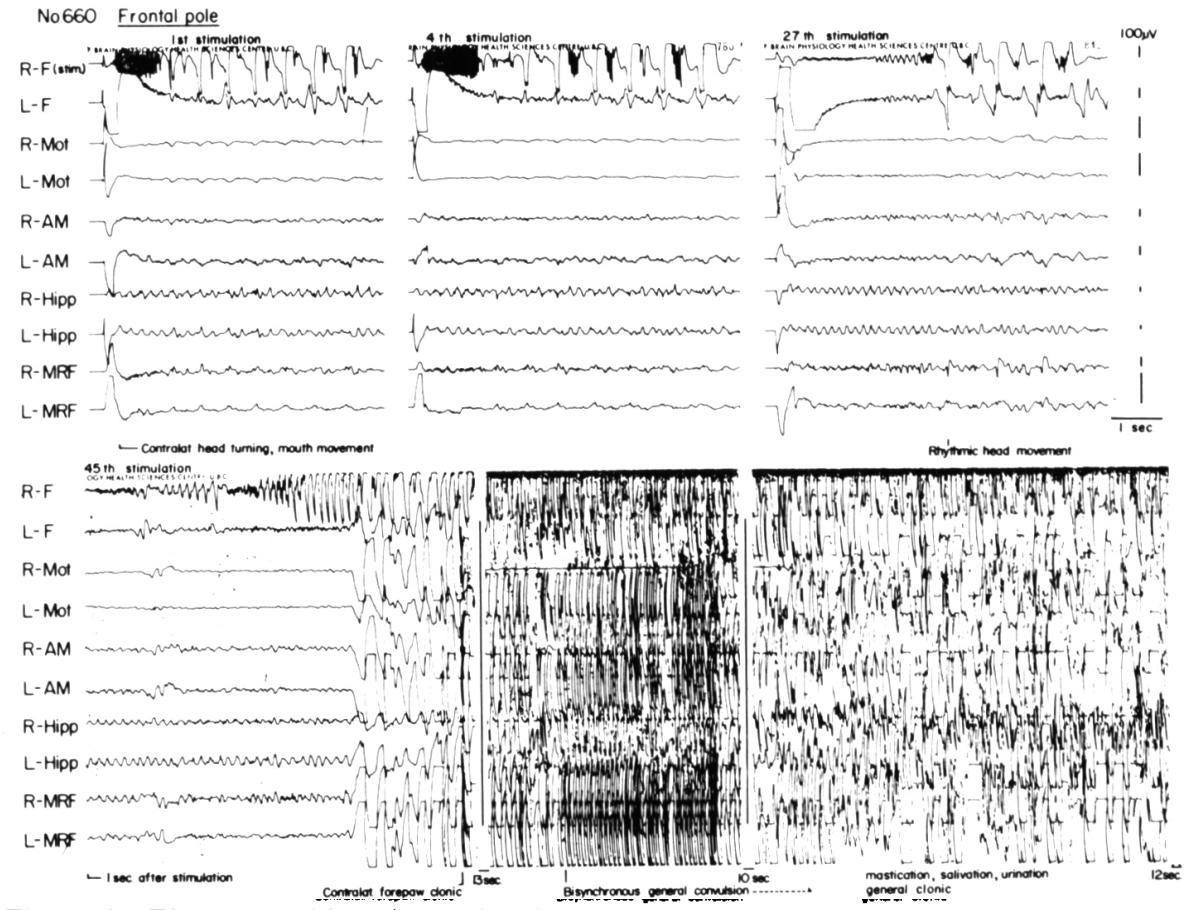

Figure 3-Electrographic seizure development of prefrontal animal. Note preferential propagation into contralateral homologous cortical area (upper row). Also note rather abrupt generalized onset of the final Stage 5 seizure (bottom row). However, EEG findings are largely artefactural.

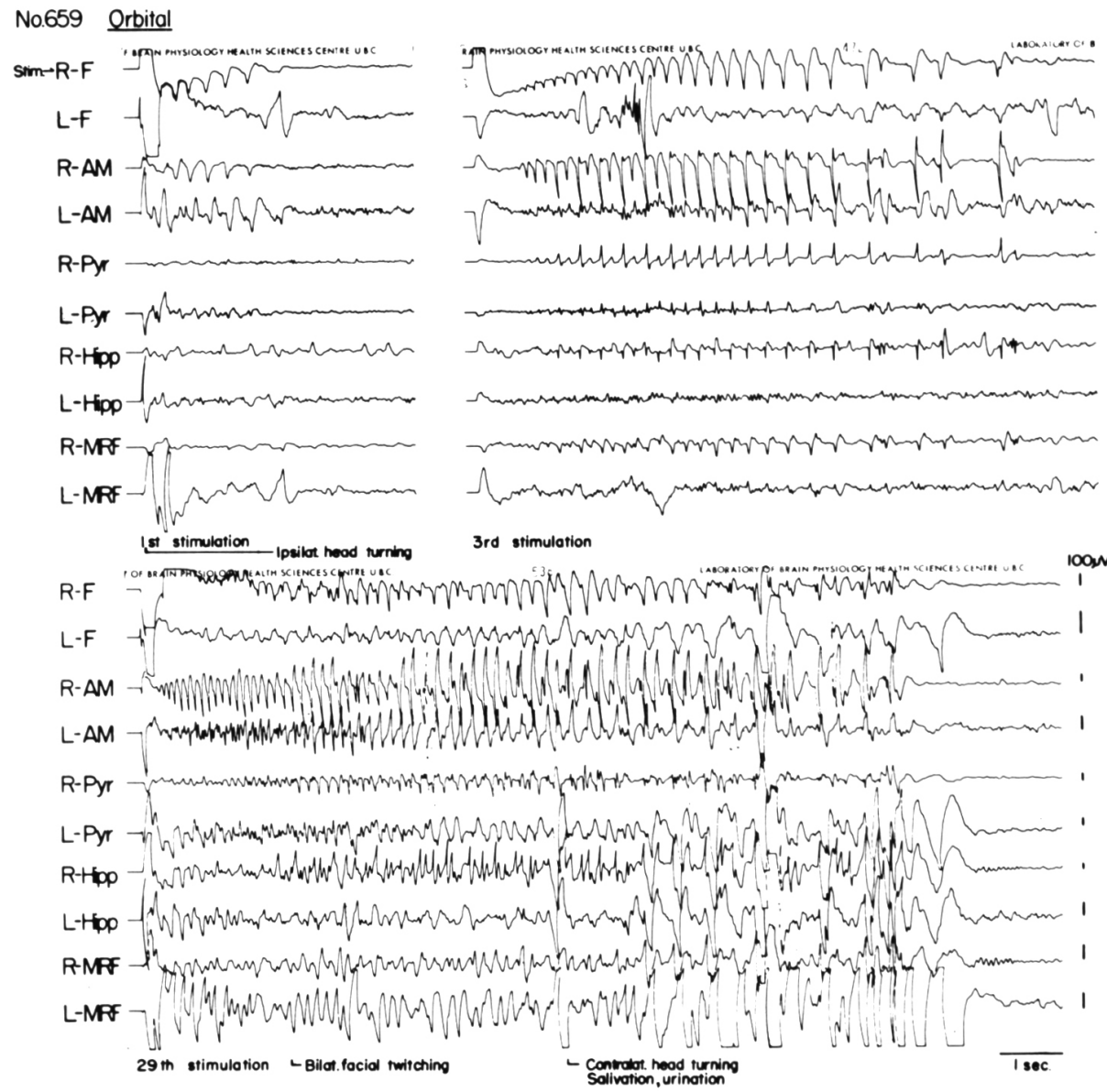

Figure 4-Ictal propagation of orbital animal. Note primarily subcortical direction of propagations. 
The configuration of the IID was mainly sharp slow wave, at times interlocked with a small spike component. With repetition of stimulation there was an emergence of similar but independent IID in the contralateral hemisphere. Such IID was rare while the animal was awake but was markedly accentuated during drowsiness and light sleep. The IID was localized at the stimulated and contralateral homologous cortical areas during the seizure development. However, once the stable Stage 5 seizure state was reached, moderate or low amplitude IID with biphasic spikes or polyspikes began to emerge in wide deep structures.

The only exception to the above was the orbital group which showed no SAD or IID at the cortical sites but developed IID of spike or polyspike configuration from the ipsilateral AM with subsequent widespread subcortical propagation beginning at Stage 3.

\section{DISCUSSION}

Results of this study show that the cat can be kindled from frontal cortical sites. Among four cortical areas examined, the orbital site showed unique patterns of seizure development strikingly reminiscent of $\mathrm{AM}$ kindling. Our finding in this group confirms earlier descriptions of behavioral and autonomic manifestations following electrical stimulation of the orbital cortex in cats (Akert, 1964; Hess and Akert, 1952). Similarity of chronological electroclinical manifestation thus observed between orbital and AM kindling suggests that they share common neurophysiological mechanisms. This is not surprising since close anatomical linkage between the orbital cortex and AM has been established (Nauta, 1962; Leichnetz, 1975; Mizuno, 1968). On the other hand, the pattern of seizure development in other cortical sites was significantly different from that of AM kindling. Thus, unlike the AM kindling, AD propagation was mainly limited to the contralateral homologous cortical sites with brief $A D$ duration. The latter showed an explosive growth when the final Stage 5 was reached which was coincident with the widespread AD propagation into deep structures. The pattern of the final stage generalized convulsion in frontal animals showed prominent tonic flexion in contrast to tonic extension observed in AM kindling. Similarly, postictal behavior pattern was characterized by prolonged and marked confusion in frontal animals in contrast to brief comatose inactivity with rather rapid recovery in AM animals. The different nature and mechanisms underlying the kindled convulsions resulting from stimulation of the frontal cortex and AM are

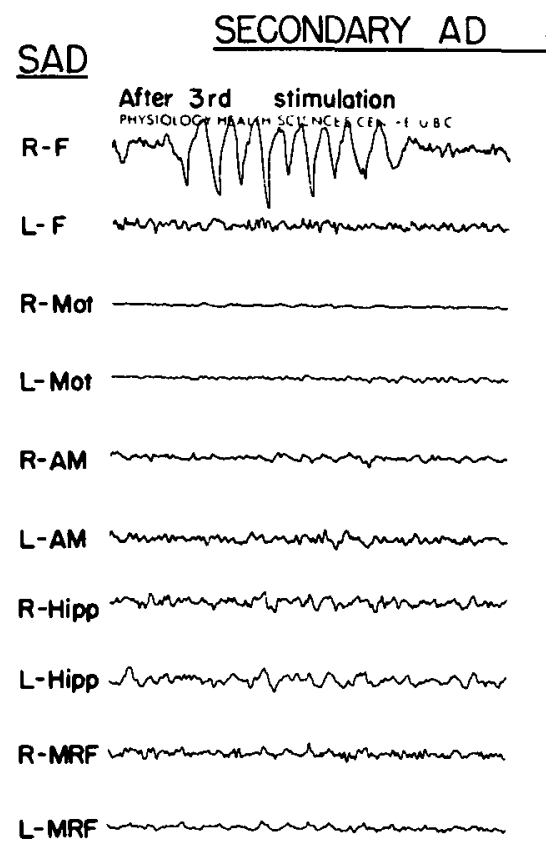

$\underline{110}$

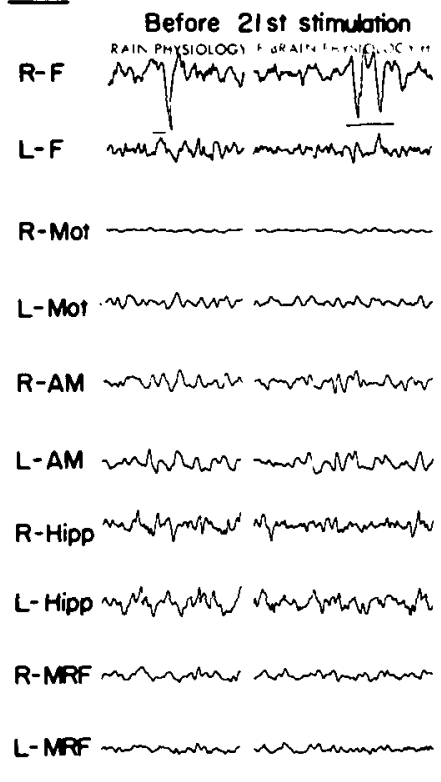

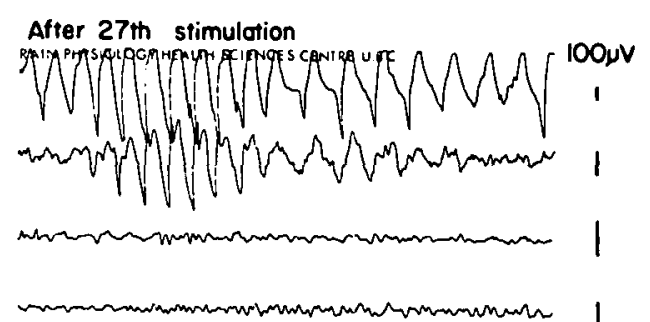

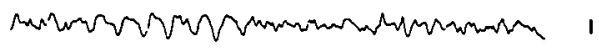
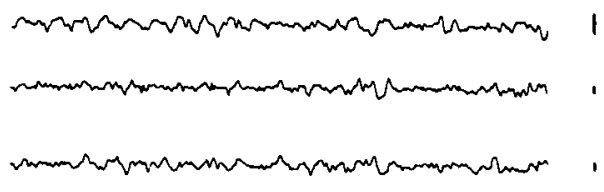

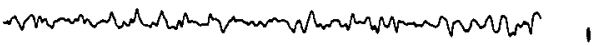

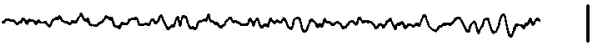
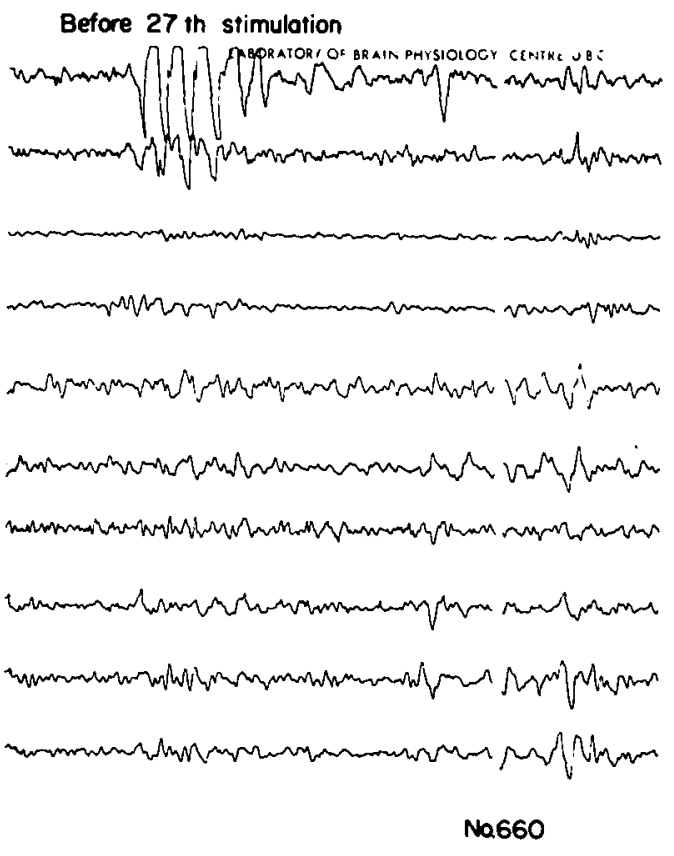

Figure 5-Secondary afterdischarge (top row) and interictal discharge (bottom row). Note exclusively cortical localization. 
further suggested by the fact that in spite of the larger average number of daily stimulations required in frontal animals (average 36 days against 25 days in AM) to reach the final seizure stage, the generalized convulsion thus induced was quite fragile in addition to their GST lacking the property of "all or none" in frontal animals.

The above findings all suggest that although frontal kindling results in minor motor manifestation from the outset, the cortical site does not have a ready access to the neuronal mechanisms underlying generalized convulsion presumably integrated in the lower brain stem. Unlike AM kindling, we have not observed electrographic evidence of early and progressive ictal participation of midbrain reticular formation in frontal kindling. Delayed, but rather abrupt, generalization and preservation of vital functions following final Stage 5 seizure in frontal group suggests its primary site of epileptogenic dysfunctions to be in the rostral brain structures. Significant modulation of the pattern of frontal kindling by sleep with marked IID accentuation and increased Stage 5 seizure stability is not inconsistent with such a view. Prominent corticofugal projection into thalamic reticular system presumably involved in the mechanism of directed attention has been demonstrated in the past (Stratford, 1954; Jasper and AjmoneMarsan, 1952; Kaada, 1960). Thus, different ictal and postictal manifestations between AM and frontal kindling may be accounted for by the difference of level of neuraxis where the primary epileptogenic dysfunction takes place.

Certain differences exist between our observation and the description of anterior neocortical kindling in rats (Racine, 1975). For example, there is a lack of AD growth even after the animal reached the generalized convulsive seizure state; the pattern of the convulsion is tonic extension instead of tonic flexion; and $A D$ threshold is generally higher than that of $A M$ in rats, to mention a few. Whether these differences represent procedural or species difference is not clear. However, enough common features exist between rats and cats and these features clearly differentiate themselves from that originating from AM in both species. Therefore, it is reasonable to conclude that the kindling process arising from the frontal lobe and that from the AM are largely independent from each other. Previous electrographic observation of early frontal cortical participation in AM kindling in rabbits (Tanaka, 1972), in cats and baboons (Wada, Sato and McCaughran, 1974), potentiation of frontal cortical response by $\mathrm{AM}$ stimulation following AM kindling (Racine, 1972) and the results of our ongoing cortical lesion study all support our concept that frontal lobe participates in but is not essential for the amygdaloid seizure development.

Clear cut differences in the chronological and spatial pattern of $A D$ and IID dissemination in frontal and AM kindling and acquisition of generalized convulsive stability following active ictal participation of limbic structure suggests progressive nature of epileptogenic dysfunction which would require mobilization of powerful subcortical neuronal network in which AM appears to be most strategically situated.

Finally, striking similarity in the pattern of electroclinical seizure development between $\mathrm{AM}$ and frontal animals emphasizes the importance of increased clinical awareness that seizure manifestations with all the landmarks of antero-mesial temporal lobe involvement may well have its origin in the basal frontal area. Indeed, such a clinical possibility has recently been summarized (Ludwig, Ajmone-Marsan and Van Buren, 1975; Geier, Bancaud, Talairach, Bonis, Szikla and Enjielvin, 1975).

Discussion of this paper follows the paper of Corcoran et al.

\section{REFERENCES}

AKERK, K. (1964). Comparative anatomy of frontal cortex and thalamo frontal connections. In J. M. Warren and K. Akert (Eds.) The Frontal Granular Cortex, 372-394, McGraw-Hill, New York.

CORCORAN, M. E., McCAUGHRAN, J. A. and WADA, J. A. (1973) Acute antiepileptic effects of $\Delta^{\text {s-tetrahydrocannabinol in }}$ Rats with Kindled seizures. Experimental Neurology, 40, 471-483.
DELGADO, J. M. R. and SEVILANO, M (1961). Evolution of repeated hippocampal seizures in the cat. Electroencephalography and Clinical Neurophysiology, 13, 722-733.

GEIER, S., BANCAUD, J., TALAIRACH, J., BONIS, A., SZIKLA, G. and ENJELVIN, M. Clinical Note: Clinical and TeleStereo-EEG Findings in a Patient with Psychomotor Seizures. Epilepsia, 16, 119-125.

GODDARD, G. V. (1967). Development of epileptic seizures through brain stimulation at low intensity. Nature, 214, 1020-1021.

GODDARD, G. V., McINTYRE, D. C. and LEECH, C. K. (1969). A permanent change in brain function resulting from daily electrical stimulation. Experimental Neurology, 25, 295-330.

HESS, R. W., AKERT, K. and MCDONALD, D. A. (1952). Function of the orbital gyrus of cats. Brain, 75, 244-259.

JASPER, H. H., AJMONE-MARSAN, C. and STOLL, J. (1952). Corticofugal projections to the brainstem. A.M.A. Archives of Neurology and Psychiatry, 67, 155-166.

KAADA, B. R. and JOHANNESSEN, N. B. (1960). Generalized electrocortical activation by cortical stimulation in the cat. Electroencephalography and Clinical Neurophysiology, 12, 573-576.

LEICHNETZ, G. R, and ASTRUC, J. (1975). Efferent connections of the orbitofrontal cortex in the marmoset. Brain Research, 84, 169-180.

LUDWIG, B., AJMONE-MARSAN, C. and VAN BUREN, J. (1975). Cerebral seizures of probable orbitofrontal origin. Epilepsia, $16,141-158$.

MIZUNO, N., SAUERLAUD, E. K. and CLEMENTE, C. D. (1968). Projections from the orbital gyrus in the cat. I. To brain stem structures. Journal of Comparative Neurology, 133, 463-476.

MORELL, F. (1973). Goddard's kindling phenomenon: a new model of the "mirror focus". In Chemical Modulation of Brain Function. Raven, New York.

NAUTA, W. J. H. (1962). Neural associations of the amygdaloid complex in the monkey. Brain, 85, 505-520.

RACINE, R. J. (1972). Modification of seizure activity by electrical stimulations. I. afterdischarge threshold. Electroencephalography and Clinical Neurophysiology, 32, 269-279.

RACINE, R. J. (1972). Modification of seizure activity by electrical stimulations. II motor seizures. Electroencephalography and Clinical Neurophysiology, 32, 281-294.

RACINE, R. J. (1975). Modification of seizure activity by electrical stimulation: cortical areas. Electroencephalography and Clinical Neurophysiology, 38, 1-12.

RACINE, R. J., GARTNER, J. G and BURNHAM, W. M. (1972). Ep:leptiform activity and neural plasticity in limbic structures. Brain Research, 47, 262-268.

STRATFORD, J. (1954). Corticothalamic connections from gyrus proreus and first 
and second sensory areas of the cat. Journal of Comparative Neurology, 100, 1-14.

TANAKA, A. (1972). Progressive changes of behavioral and electroencephalographic responses to daily amygdaloid stimulations in rabbits. Fukuoka Medical Journal, 62, 152-164.

WADA, J. A, and SATO, M. (1974). Generalized convulsive seizures induced by daily electrical stimulation of the amygdala in cats. Neurology, 24, 565-574.
WADA, J. A. and SATO, M. (In Press). The generalized convulsive seizure state induced by daily electrical stimulation of the amygdala in split brain cats. Epilepsia.

WADA, J. A. (In Press). Progressive seizure development in subhuman primates and effect of cerebellar stimulation upon developed VS. developing amygdaloid seizures. In. A. Escobar (Ed.) Xth Anniversary Volume, National Institute of Neurology, Mexico City.
WADA, J. A. and OSAWA, T. (In Press). Generalized convulsive seizure state induced by daily electrical stimulation in Senegalese baboon, Papio papio. Neurology.

WADA, J. A., SATO, M. and McCAUGHRAN, J. A. (1974) Cortical electrographic correlates of amygdaloid seizure development in rats, cats and baboons. Presented at Annual Meeting of Eastern EEG Association. December 4, 1974, New York. 